\title{
TomoSim: a tomographic simulator for DOAS
}

\author{
Rui Valente de Almeida ${ }^{1,2}$, Nuno Matela ${ }^{3}$, and Pedro Vieira ${ }^{1}$ \\ ${ }^{1}$ Physics Department, FCT NOVA, Caparica, Portugal \\ ${ }^{2}$ Compta SA, Lisbon, Portugal \\ ${ }^{3}$ University of Lisbon - Biomedical Engineering and Biophysics Institute \\ Correspondence: Rui Valente de Almeida (rf.almeida@campus.fct.unl.pt)
}

\begin{abstract}
.
TomoSim comes as part of project ATMOS, a miniaturised DOAS tomographic atmospheric evaluation device, designed to fit a small drone. During the development of the project, it became necessary to write a simulation tool for system validation. TomoSim is the answer to this problem. The software has two main goals: to mathematically validate the tomographic acqui-

5 sition method; and to allow some adjustments to the system before reaching final product stages. This measurement strategy was based on a drone performing a sequential trajectory and gathering projections arranged in fan beams, before using some classical tomographic methods to reconstruct a spectral image. The team tested three different reconstruction algorithms, all of which were able to produce an image, validating the team's initial assumptions regarding the trajectory and acquisition strategy. All algorithms were assessed on their computational performance and their ability for reconstructing spectral "images", using two phantoms, one of which custom made for this purpose. In the end, the team was also able to uncover certain limitations of the TomoSim approach that should be addressed before the final stages of the system.
\end{abstract}

\section{Introduction}

TomoSim was born as a software platform for the ATmosphere MOnitoring System (ATMOS) project, which was a Portuguese EU funded project that aimed to develop a miniaturised spectroscopy system for atmospheric analysis and trace gas mapping using Differential Optical Absorption Spectroscopy (DOAS), with tomographic capabilities. FCT NOVA participated in this project as part of a consortium which also included Compta, one of the oldest IT groups in Portugal.

The ATMOS project required the development of an actual physical system, but before that stage is reached, it became necessary to develop a simulation software platform. This simulating application had to be designed while taking the ATMOS project's specifications into account: simulations had to be similar to what the project's final intended capabilities will allow. Otherwise, it would not be possible to make any predictions regarding the physical system and namely its reconstruction limitations. By writing the simulator, the researchers had the goals of validating their acquisition strategy and to help the engineering team anticipating any threats that might come from this part of the project. 
The ATMOS system's reconstruction method is predicated on the assumption that, in low optical densities and with circular trajectories of up to $1 \mathrm{~km}$ in diameter, it should be possible to produce a fan beam array of target trace gases concentrations using a drone and programming it to perform a series of carefully programmed sequential spectral measurements. Column density values retrieved by the device would then be used as projections, which could then be used to tomographically reconstruct an "image". This image would in fact correspond to the geographic distribution of the target trace gases. This same assumption is shared with the simulator, which makes use of Python (Perez and Granger, 2007) and some of its main libraries, like NumPy (Oliphant, 2007) to reconstruct the said "image" using three different algorithms: fan beam Filtered BackProjection (FBP), Simultaneous algebraic Reconstruction Technique (SART) and Maximum Likelihood Expectation Maximisation (MLEM). Two tomographic phantoms were used: Shepp-Logan's standard medical imaging phantom, and one custom-made phantom for DOAS spectral measurements. All three mathematical algorithms managed to reconstruct the image. FBP was shown to be significantly more cost-effective than the other two. The custom coded MLEM routine was not on-par with the other two solutions, which were plugged directly from the SciPy library.

Besides runtimes and other significant software engineering considerations, the development of TomoSim concluded that the assumptions of the team regarding the acquisition strategy for spectral geographic images were correct, but lifted the veil on some limitations that should be addressed before building the final system.

The paper is structured as follows: the section after this introduction, Section 2, targets the theoretical background with which the paper was built; Section 3 describes the design, the rationale behind it and the technical choices that have been taken in the making of TomoSim; in Section 4 there is a description of the results that were obtained through the simulator, including reconstructions, running times and Section 5 is dedicated to the conclusions that were taken using this piece of software, as well as some foreseeable future developments.

\section{Theoretical Background}

This section explains the two main theoretical frameworks upon which the tomographic simulator was built: DOAS and Tomography. The authors do not wish to make this section a comprehensive description of any one of these theories, but the following should be sufficient to introduce the reader to both subjects.

\subsection{DOAS}

DOAS is a widely used atmospherical analysis technique, developed in the late 1960s. Fundamentally, it is an absorption spectroscopy technique, therefore based on Lambert-Beer's law. This law was actually first formulated by Pierre Bouguer in 1729. At the time, he wrote that "in a medium of uniform transparency, the light remaining in a collimated beam is an exponential function of the length of the path in the medium" (APS, 2011). This theory can thus be written as in Equation 1. 
In which $I_{0}(\lambda)$ is the source intensity of a light beam, $I(\lambda)$ the intensity of the light that reaches the detector, $\sigma$ is the cross-section of the chemical compound being measured, $c$ its concentration and $L$ the optical path of the light. Finally, $\lambda$ is the wavelength of the radiation.

The rearrangement of Equation 1 gives us the concentration, and more importantly, allows us the creation of a new quantity,

5 D, which is called optical depth. This is explicit in Equation 2 (Struve, 1989; Platt and Stutz, 2007).

$c=\frac{\ln \left(\frac{I_{0}(\lambda)}{I(\lambda)}\right)}{\sigma(\lambda) \cdot L}=\frac{D}{\sigma(\lambda) \cdot L}$

In the laboratory, this equation can be (and is) used directly and with few obstacles, since there is very little variability, and that which exists is controlled for. In the open atmosphere, this is not the case at all.

There are many factors that influence the degree to which we can apply Lambert-Beer's rule in atmospheric measurements.

10 The most important of which is the fact that in order to know the source intensity $\left(I_{0}(\lambda)\right)$, one would have to remove any and every absorber from optical path, and this is clearly unfeasible in the great majority of circumstances. To surpass this problem, DOAS measures the difference in absorption at two different wavelengths. The principle has been in use since the 1930s, when Dobson used his own spectrometer to measure the total column of atmospheric ozone.

There are many different DOAS systems, but the single greatest distinction among them is whether they are active or passive. Active systems use artificial light sources such as a Xenon lamp to measure chemicals in the air. Passive systems use natural sources, such as the Sun or the Moon (or other celestial bodies). This paper will focus on passive systems, since the ATMOS project is a scattered sunlight DOAS system, and thus passive.

Scattered sunlight DOAS systems have their own specific particularities and special properties. For instance, the optical path is unknown. It does not use direct sunlight, so there was scattering, but exactly where this takes place cannot be determined a priori. Scattering also implies that there is a fraction of the source's light that does not reach the detector. Besides these, one must account for all the other passive DOAS common effects, such as the fact that there are many absorbers that have spectral structures overlapping the target species; that there are Fraunhofer lines, Mie and Rayleigh scattering, turbulence and instrumental effects, etc.

Another particularity of scattered DOAS is that the light path is not known.

All these influential effects mean that, in the open atmosphere, Equation 3 is a more plausible model than that presented in Equation 1.

$$
\begin{aligned}
I(\lambda)=I_{0}(\lambda) & \cdot A(\lambda, \ldots) \cdot \\
\cdot & \exp \left[-\int\left(\sum_{i} \sigma_{i}(\lambda, s) .\right.\right. \\
\cdot & \left.\left.c_{i}+\epsilon_{M}(\lambda, s)+\epsilon_{R}(\lambda, s)\right) d s\right]
\end{aligned}
$$


In Equation 3, there is more than one absorber, which is denoted by index $i$ in the sum. $A(\lambda, \ldots)$ denotes the fraction of light that gets scattered into the detector, $\epsilon_{M}$ and $\epsilon_{R}$ are Mie and Rayleigh scattering coefficients, and the integral is performed on the whole optical path, $s$.

Typically, we measure a trace gas's atmospheric contribution by its total column. This quantity is essentially the integral of

5 the compound's number density, in molecules $/ \mathrm{cm}^{3}$, over a column that goes from the ground to the Top Of the Atmosphere (TOA). In Equation 4, the number density for molecule $x$ is denoted $c_{x}$.

$C_{x}=\int_{0}^{T O A} c_{x}(z) d z$

One can look again at Equation 3 with the interest of quantifying only the trace gas contribution. This contribution should be somehow related to the total column, since the only real difference between what the DOAS instruments see and the total column is the dependence on the optical path of the former. In fact, we can separate the integral to get the trace absorbers optical density expression, displayed in Equation 5. Since we can consider the cross-section to be fairly constant with the optical path, it is possible to separate this integral, and we arrive at the very important value of the slant column, $S C_{i}$, which is the integral of the gas's number density along the optical path.

$S C_{i}=\int c_{i}(s) d s$

The integration of the absorption coefficients, which are inside the exponential term of Equation 3 to their optical densities, we arrive at the expression in Equation 6. This equation cannot be solved without some very seldomly available data, such as the original light source $I_{0}$. DOAS allows us to overcome this problem by making relative measurements (hence the differential in the name of the technique), using another scattered light spectrum as reference and by observing that most interesting trace gases have very narrow spectral structures, while effects like Mie and Rayleigh scattering have broad spectral features.

$\ln \left(\frac{I_{r e f}}{I}(\lambda)\right)=\ln \left(\frac{A_{r e f}}{A}\right)+\sum_{i} \sigma_{i}(\lambda) \cdot \Delta S C_{i}+\tau_{R}(\lambda)+\tau_{M}(\lambda)$

With this in mind, it is possible to separate the original optical depth in two parts, a differential part, which is comprised of the narrowband contributions coming mainly from trace gases; and the "continuous" part, which contains the slow-variation, broadband contributions in Equation 3. The latter can be approximated by fitting a low order polynomial to the original optical depth signal. If one subtracts this polynomial from the optical depth, the result is the narrowband signal that constitutes the differential part. Target absorbers column densities can then be found by fitting their cross-sections to the differential signal, using a numerical algorithm such as Levenberg-Marquardt's (Press et al., 2007).

This small introduction to the topic of DOAS is more than enough to explain and understand the rest of this article regarding the spectroscopic procedures. References (Platt and Stutz, 2007; Merlaud, 2013) contain a more thorough coverage of the topic, both with reference to the technique's physics and instrumental considerations. 


\subsection{The Tomography Problem}

\subsubsection{Introduction}

Tomography is the cross-sectional imaging of an object through the use of transmitted or reflected waves, collected by the object exposure to the waves from a set of known angles. Tomography has many different applications in science, industry, and 5 in particular, medicine. Since the invention of the Computed Tomography (CT) machine, in 1972 by Hounsfield (Gunderman, 2006), tomographic imaging techniques have had a revolutionary impact, allowing doctors to see inside their patients without having to subject them to surgical interventions. Examples of tomographic applications for scientific and industrial purposes include radio-astronomy and certain kinds of tomographic non-destructive test techniques (Kak and Slaney, 2001).

Tomography has had its mathematical basis set by Johannes Radon, a German mathematician that proved that it is possible to represent a function in $\mathbf{R}$ in the space of straight lines $\mathbf{L}$ through its line integrals. A line integral is an integral in which the function that is integrated is evaluated along a curved path. In the tomographic case, these integrals represent a measurement on a ray that is traversing the field of analysis. Each set of line integrals (rays), characterised by a given projection angle, is called a projection. The set of all projections, arranged in matrix form by projection angle and detector is called a sinogram. All reconstruction methods revolve around this matrix and the data it contains (Bruyant, 2002; Kak and Slaney, 2001; Herman, 1995).

\subsubsection{Discretisation}

One of the first steps one has to undertake when approaching real-world problems in a computational way is to discretise the analysis field, since nature presents itself in a continuous manner, but computers only work in discrete values. There is not a single answer when dealing with this subject in tomographic studies, all with their drawbacks and advantages. In this particular study, Siddon's algorithm was chosen for this purpose, as it is one of the most reliable and studied ways of performing this task.

The algorithm is based on the consideration that each pixel is the intersection area of orthogonal sets of equally spaced parallel planes (or lines, in two dimensions), and that it is possible to parameterise a line integral according to this idea, with the ray being written (for two dimensions):

$X(\alpha)=X_{1}+\alpha\left(X_{2}-X_{1}\right)$

$25 Y(\alpha)=Y_{1}+\alpha\left(Y_{2}-Y_{1}\right)$

In Equation 7, $X_{1}$ and $Y_{1}$ are the coordinates for the entry point (of the ray in the analysis field) and $X_{2}$ and $Y_{2}$ are the exit point coordinates. $\alpha$ is the parametric value. If the ray is totally contained within the field of analysis, this value varies between 0 and 1 ; otherwise, it has its minimum at the entry point and maximum at the exit.

The parametrical representation of the line integral, allows one to recursively calculate all intersections between the ray and the grid defined by the orthogonal lines described above. The differences between intersection points render the lengths of 
each ray contained within each pixel. The sum of all the lengths for one ray yields the total value of the line integral, which corresponds to a projection. The algorithm steps are presented in Algorithm 1 (Siddon, 1985; Gao, 2012).

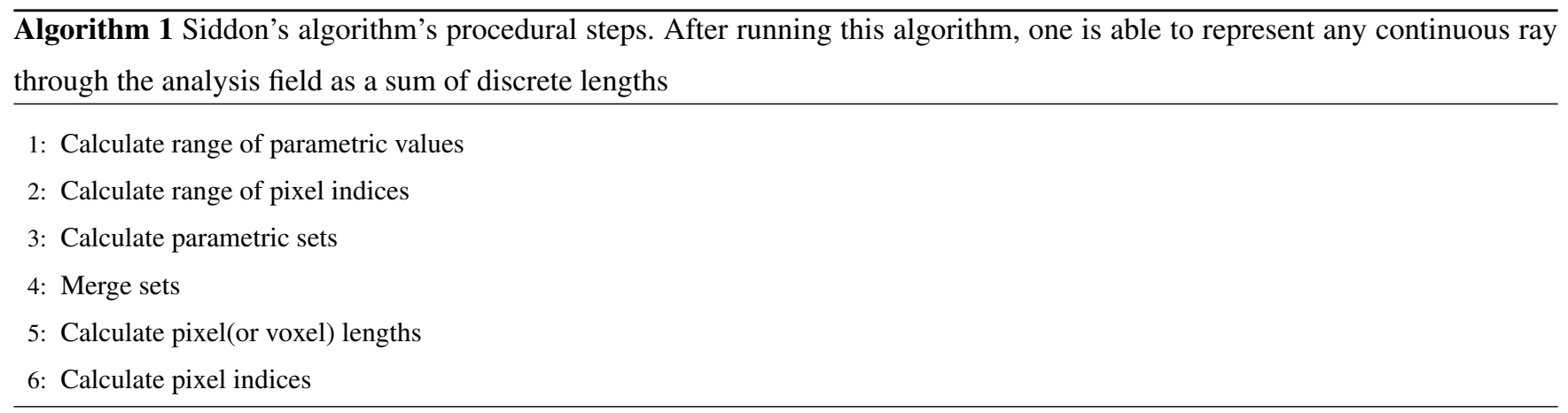

\subsubsection{Geometry}

The application of analytical algorithms such as FBP depends on the type of geometric arrangement of the tomographic 5 problem. TomoSim uses two tomographic geometries in its reconstructions: parallel and fan beam.

Parallel Projection geometry (see Figure 2) is the most basic assembly. In this arrangement, each projection is a set of parallel line integrals, as can be seen in Figure 2. The radiation sources can be assembled in a linear array, facing detectors in the same number on the opposite side of the target object, or, in alternative, a single source can move in a linear trajectory, directing its rays onto an array of detectors, linearly arranged on the opposite side of the target object. Projections of this sort are characterised by the projection angle, which is the angle each ray makes with the vertical axis. The other relevant geometric assembly is the fan beam arrangement (see Figure 1). In this projection mode, all radiation in the projection comes from a single source, with rays being directed outwards onto a set of detectors, which may be arranged on a circumference arc (equiangular rays) or on a straight line (equally spaced rays) (Kak and Slaney, 2001).

\subsubsection{Reconstruction}

15 The overarching division between tomographic reconstruction algorithms is on the level of their nature, which can be analytical or algebraic (iterative). Other subdivisions come from the geometry and the type of technology used for the particular application on which reconstruction is being run. In medical imaging, the most common analytical method is the Filtered BackProjection algorithm (FBP). FBP is based on Fourier's Slice Theorem, which states that the one dimensional Fourier Transform (FT) of a projection at a given angle is the two dimensional FT of the reconstructed image through that same angle.

20 If a sufficient number of projections is gathered, one can create a good estimate of the image by performing a 1D FT of the projections, and inverting them in 2D, before summing them in the image space. It so happens that this direct inversion process produces heavily distorted images. FBP overcomes this problem by introducing a weighing filter, which is applied to each projection before inversion. 


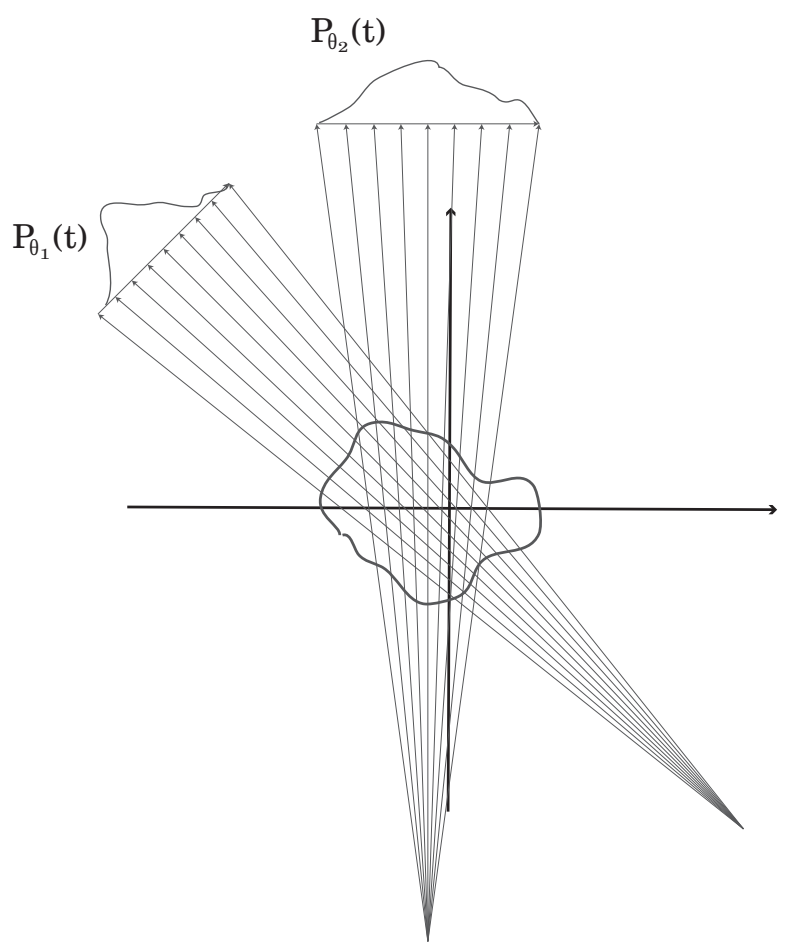

Figure 1. Fan beam projection geometry schematic, depicting equidistant sensor positions.

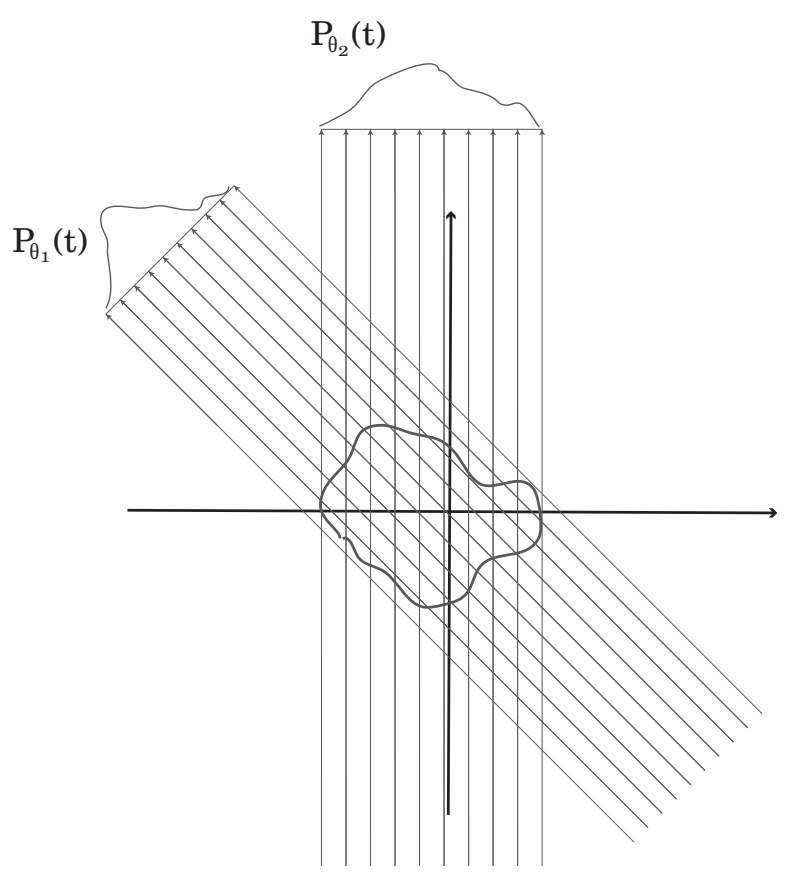

Figure 2. Parallel projection geometry.

This sequence of steps is sufficient for parallel projection reconstruction, but for fan beam projections, the FBP can only be applied after a series of somewhat cumbersome geometric transformations. If this is not acceptable, for some reason, there is an alternate solution: the fan beam sinogram can be reorganised, based on the observation that a ray in a fan beam is equal to a ray in a parallel projection in another given angle. Of course this resorting operation will not render a perfect sinogram for this new parallel projection (since not every fan ray has a direct parallel ray correspondent), but imperfections can be normally corrected through interpolation. After this procedure is run, one can proceed as if the geometry were parallel (Kak and Slaney, 2001; Herman, 1995; Defrise et al., 2005).

Iterative algorithms are based on simpler premises, but require a different mindset. The set of projections can be thought of as a matrix, called sinogram, as has been introduced in this same section. In this matrix, the lines refer to the projection number, and the columns deal with the detectors (for instance, the first line of this matrix corresponds to all detections in the first projection). The image can also be thought of as a matrix, in which each pixel has a given value, which gives it its intensity (and/or colour). Finally, there is the system matrix, which is the matrix that contains the lengths of every ray in each projection contained in each one of the image's pixels, lengths which are obtained, in this case, through Siddon's algorithm, already presented. Iterative algorithms, in general, attempt to solve Equation 8 . In it, $g \in \mathbb{R}^{m, 1}$ is the column vector sinogram, $\mathbf{a} \in \mathbb{R}^{m, n}$ is the system matrix and $\mathbf{f} \in \mathbb{R}^{n, 1}$ is the column vector image. $m$ is the number of measurements (projections times 
detectors) and $n$ is the number of pixels in the image. As their designation implies, iterative algorithms produce an estimation for $\mathbf{f}$ which is updated in the direction of error minimisation in every iteration (Kak and Slaney, 2001; Bruyant, 2002).

$\mathrm{g}=\mathbf{a} \cdot \mathbf{f}$

The popularity of algebraic reconstruction methods has not remained constant throughout the years. For a long time, they 5 have been considered too computationaly intensive to use in a clinical setting (paradoxically, Hounsfield's machine used this kind of algorithm). This was in direct opposition to the fact that researchers know that these methods are better able to model reconstruction since Shepp and Vardi published the maximum likelihood tracer estimation in 1982. Nowadays, and since the mid nineties, these algorithms are the first choice whenever the reconstruction dataset is not exceedingly large (Defrise et al., 2005).

The general goal of iterative reconstruction algorithms is to solve Equation 8 (Bruyant, 2002). In principle, any method that solves it can be used for image reconstruction in tomography. In reality, however, only a few are currently in use by the community. Of these, TomoSim uses two of the most prominent: Simultaneous algebraic Reconstruction Technique (SART) and Maximum Likelihood Expectation Maximisation (MLEM).

SART was presented in 1984 by Andersen and Kak (Andersen, 1984) and the global idea is that the estimated image is corrected for all projections at the same time (in opposition to the original algebraic Reconstruction Technique, in which corrections were applied for each single projection). Iterations in SART change the estimated image according to Equation 9, iterating on $k$.

$\mathbf{g}_{i}^{(k+1)}=\mathbf{g}_{i}^{(k)}+\frac{\sum_{j}\left[\mathbf{a}_{i j} \cdot \frac{p_{j}-\mathbf{a}_{j}^{T} \cdot \mathbf{g}^{(k)}}{\sum_{i=1}^{n} \mathbf{a}_{i j}}\right]}{\sum_{j} \mathbf{a}_{i j}}$

MLEM algorithms were first published in the medical imaging community in 1982, by Shepp and Vardi (Shepp and Vardi, 1982). With this algorithm, image corrections are ruled by Equation 10, which also iterates over $k$.

$\mathbf{f}_{j}^{k+1}=\frac{\mathbf{f}_{j}^{k}}{\sum_{i=1}^{n} \mathbf{a}_{i j}} \sum_{i=1}^{n} \frac{\mathbf{g}_{i}}{\sum_{j^{\prime}=1}^{m} \mathbf{a}_{i j^{\prime}} \mathbf{f}_{j^{\prime}}^{k}}$

This equation is very easy to implement computationally, if one observes that the sums of the second multiplication term expand neatly onto matrix products. In the end, this equation is the equivalent of writing Equation 11, as explained in (Bruyant, 2002), in which $\mathrm{IMG}^{(k)}$ is the estimated image in the $\mathrm{k}^{\text {th }}$ iteration, NBP is the Normalised Backprojection operation, RSNG the real sinogram (as in coming from the detector hardware) and SSNG the simulated sinogram, calculated through the previous iteration.

$$
\mathrm{IMG}^{(k+1)}=\mathrm{IMG}^{(k)} \times \mathrm{NBP}\left(\frac{\mathrm{RSNG}}{\mathrm{SSNG}^{(k)}}\right)
$$




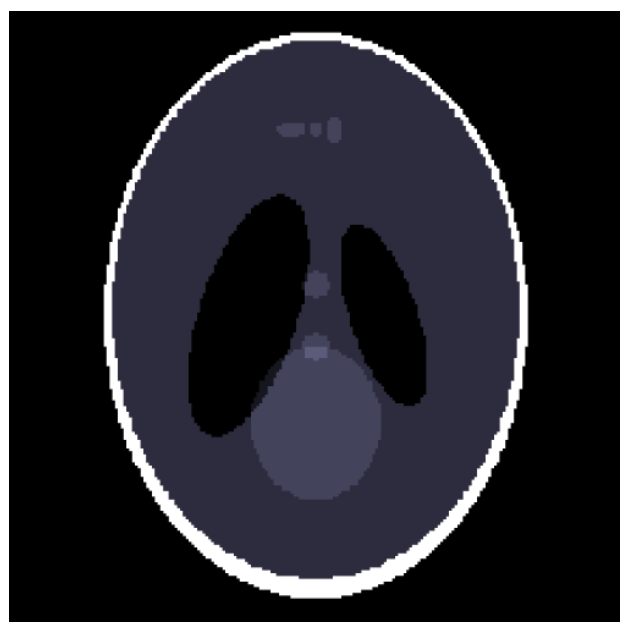

Figure 3. Shepp-Logan phantom, commonly used in medical imaging.

\subsection{The Shepp-Logan Phantom}

In medicine, a phantom is a model that emulates certain properties of human or animal tissue, which is used for the evaluation of a given diagnostic or therapeutic method. They are used widely in medical imaging, allowing researchers to analyse the performance and calibrate their algorithms. In computed tomography, the Shepp-Logan phantom is one of the most commonly used phantoms in existence.

I their 1974 paper (Shepp and Logan, 1974), Larry Shepp and Benjamin Logan set out to present a Fourier-based reconstruction technique for a head section. The most famous result of this paper, however, was not their method, but rather the phantom they used in their experiments. This phantom, which became ubiquitous in medical imaging in the following decades was named the Shepp-Logan phantom. It is the graphical representation of the sum of 10 ellipses, with different center coordinates, axis and alignments. This image, is reproduced in Figure 3.

Its prominence in the medical field, where the techniques used in TomoSim have emerged, dictates the inclusion of this phantom in this study, even if it is not the perfectly adequate for the goal of atmospheric imaging through spectral projections, given that human heads and trace gas distributions have little in common. A more suitable phantom, more reflective of the gaseous nature of the analysis object, is presented in Section 3.

\subsection{DOAS Tomography}

DOAS tomography is a relatively new field of study within DOAS. It involves, as the name implies, the application of tomographic techniques to the atmospheric studies that are normally conducted through DOAS. The concentration values retrieved through the spectroscopic technique are essentially line integrals in themselves. Therefore, they can be almost immediately considered projections. If one gathers enough of these integrals from a sufficient number of angles, any tomographic algorithm is able to reconstruct an image, which corresponds to a map of concentrations of the target trace gas in the study. 
One of the first suggestions of a technique which could be adapted to the DOAS procedures was made in 1979 (Byer and Shepp, 1979). However, the first study that applied tomography to DOAS in a significant manner was the BAB-II campaign (Laepple et al., 2004; Pundt et al., 2005). This was a research initiative that involved people from the Heidelberg DOAS group and intended to study the temporal evolution of the $\mathrm{NO}_{2}$ concentration in a bi-dimensional way, along the motorway that connects Heidelberg to Mannheim, in Germany. This campaign led to several publications and is to this day the main "contributor" to tomographic studies with DOAS. More recently, in 2016, Stutz and his team have built and used a similar set-up to study the atmospheric profiles of aromatic hydrocarbons near an refinery plant, in Texas (Stutz et al., 2016). Their system was composed of a dual-light emitting diode light source, a telescope which acted as emitter and receiver of light and retro-reflector arrays, positioned strategically in the geographic region that was being studied. Although this study was not as extensive as the previously mentioned Heidelberg study, it is also very important, as it proves the practical applicability of the technique to real world problems. Finally, it is worth to mention the paper by Erna Frins, who in 2006 used sun-illuminated targets to perform a tomographic analysis of the region in which her system was positioned, which coincidentally is also Heidelberg. This study is important because it is one of the few that uses scattered sunlight with this technique. Moreover, it also features a very good description of the physics and mathematical approximations that are inherent to the experiments at hand.

These studies and more are addressed in another paper, which should be submitted shortly, and in which the authors have conducted a deeper and more systematic literature review on the subject.

\section{Methods}

\subsection{Device description}

TomoSim is a simulation platform for a drone-mounted atmospheric monitoring system based on DOAS. Although the physical device has not yet been assembled, the team has already compiled a final (or very close) design, which is schematically represented in Figure 4. The reasoning behind the custom design was to increase the maximum payload and allow longer flight times. The team chose to use a DJI S900 frame (hexacopter), with custom-made $368 \mathrm{~mm}$ carbon fibre arms, longer and lighter than the original. The increased empty space allows the replacement of the default propellers by 17 " carbon fibre units, coupled to 6 E1200 motors. This propeller-motor configuration is not only significantly more powerful than the default assembly, but are also more efficient. According to the manufacturer (DJI, 2015), this configuration is able to lift and work with payloads exceeding $8 \mathrm{~kg}$, which is much more than we need for data acquisition platform, comprised of the gimbal, a Celera SSIN-06 (Celera, 2019) unit with a maximum pointing error of 2 arcseconds; the telescope, an Omegon MightyMak (Omegon, 2019); and an Avantes Mini spectrometer with 2048 channels (Avantes, 2019).

A Pixhawk flight controller is used to handle and manage aerial dynamics, and also to gather every sensor output in the device. The controller comes with integrated gyroscopes, magnetometers and barometers. The only external sensor that needs to be adapted and purchased separately is the navigation (GPS) unit. The Pixhawk supports Real Time Kinematic GPS (RTKGPS), a combination of inertial sensors and satellite navigational data that can grant the UAV a positioning precision under 20 cm (Triantafyllou and Aspragathos, 2011; Zimmermann et al., 2017). The flight controller is in permanent communication with 


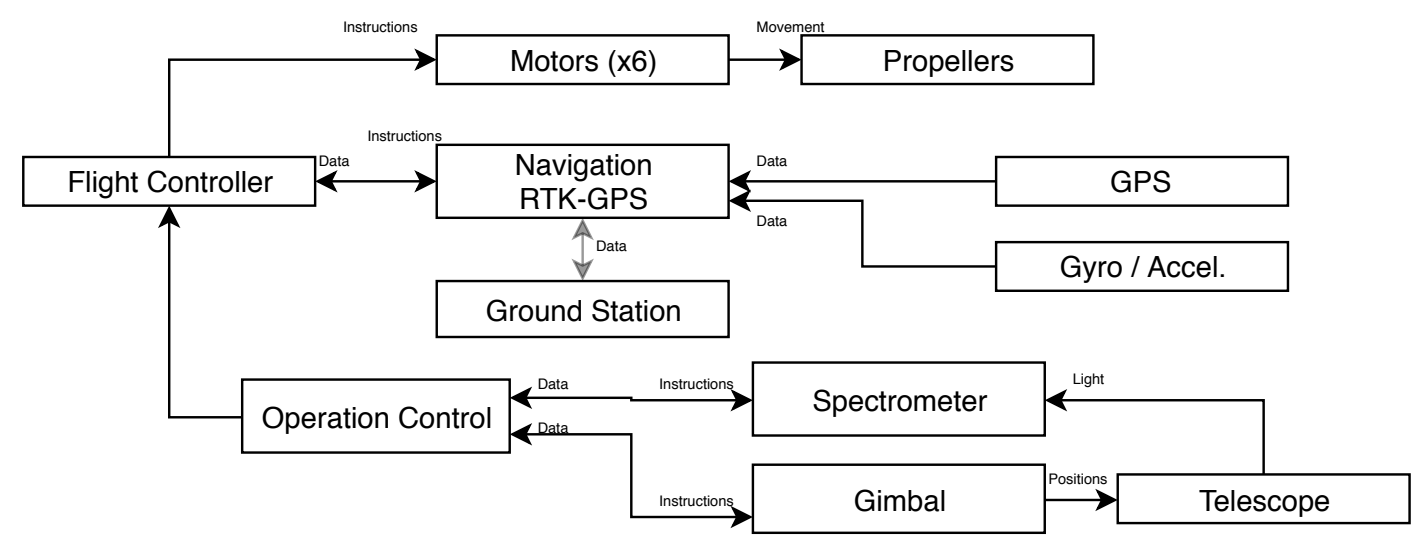

Figure 4. Drone system schematic representation, with component relations.

the operation controller, which is a Raspberry Pi 0 (or similar) single board computer. This computer stores the flight program and directs the flight controller to each necessary position, and also controls data acquisition through a USB connection to the spectrometer. The device's trajectory will be planned using Arducopter's Python libraries and their Software In The Loop (SITL) simulation platform.

\section{3.2 Data acquisition}

The simulated drone is to describe an unobstructed horizontal circular trajectory with a maximum diameter of $1 \mathrm{~km}$ at the intended measurement height, the interior of which is the Region Of Interest (ROI). There are two moments to the data gathering process.

First moment While flying in this circle, the device stops in a series of positions at a given fixed angular interval $(\Delta)$ from each other. The number of stops is defined by $\Delta$ and by fan beam information requirements (see (Kak and Slaney, 2001)) At each one of these stops, the gimbal turns towards the trajectory's interior and points in a series of angular directions that describe an arc. For procedural simplicity, the angle between these directions is also $\Delta$. At each one of these angles, the device's operational controller instructs the spectrometer to acquire a given number of spectra, which depends on configuration and conditions. Besides spectral data, the system algebraically calculates and stores the point in which the light will exit the ROI (see Appendix A). The first trajectory moment is schematically represented in Figure 5

Second moment The device positions itself in each of the points in which light has exited the ROI in the first moment and the gimbal is pointed towards the entrance point, effectively aiming in the opposite direction to which a spectral measurement took place in the $1^{\text {st }}$ moment. Light that comes from the sun is scattered somewhere in the atmosphere and enters the $\mathrm{ROI}$ (at a given angle) in point $\mathrm{A}$. It then traverses the distance $\mathrm{AB}$ and leaves the ROI in point $\mathrm{B}$. At these distances and with this kind of geometry, light scattering can be considered negligible (Casaballe et al., 2017; Kern et al., 2010; Frins et al., 2006) and therefore light extinction will primarily be due to absorption by components between A and B (Platt 
and Stutz, 2007). It should then be possible to apply Lambert-Beer's law to extract trace gases concentrations in the ROI, by considering light at point $\mathrm{A}$ as the source intensity $\left(I_{0}\right.$ in Equation 1$)$ and light at B the final intensity ( $I$ in Equation 1). When the $2^{\text {nd }}$ moment is complete, the system has a set of fan beam distributed spectra, which can be equated to projections in a tomography problem.

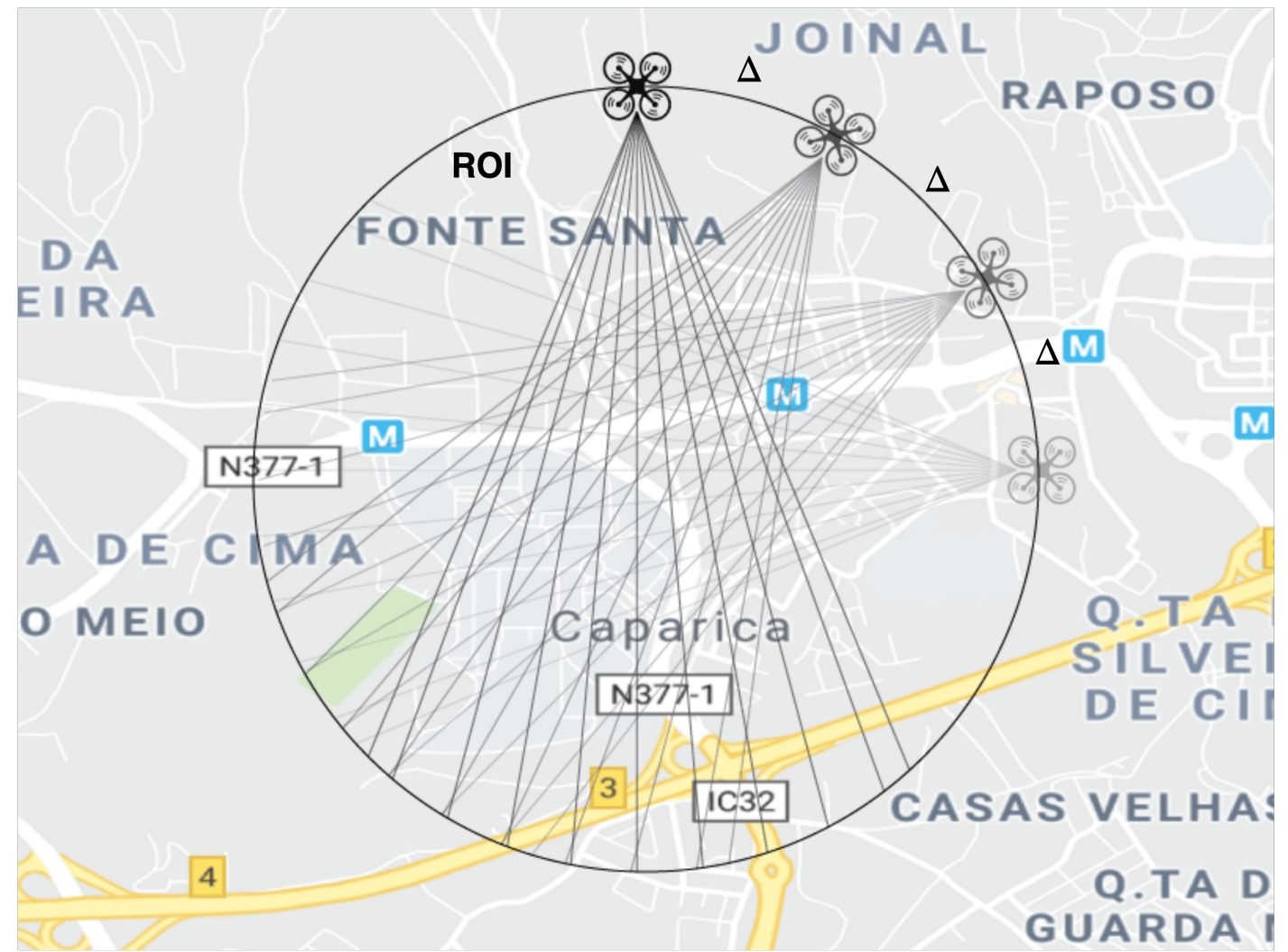

Figure 5. $1^{\text {st }}$ data acquisition moment. The drone describes a circular trajectory that limits the ROI. The angular interval between the points on which the drone stops is denoted $\Delta$. The (not represented) angular interval between rays within the fan is, in this particular experiment, also equal to $\Delta$ for reconstruction purposes. () Google Maps in 2019.

\subsection{Phantoms}

The previous two subsections have described the device that is planned to be used for the physical deployment of TomoSim and the trajectory that this device is required to perform for tomographic reconstruction of the trace gas concentration mapping in a given region. There is still one component left for the simulation to run, which is a phantom. As stated in Section 2, a phantom is a known image that is used in tomographic imaging to test inversion algorithms and their performance. In this paper, two phantoms are used. One is the Shepp-Logan phantom, already described in Section 2.3. The other one, which will be referred to as the "new spectral phantom", was designed especially for this simulator and is based on the idea that a two-dimensional (in 
this case) Gaussian peak is more appropriate to describe the smoother nature of the distribution of a gas than a series of sharply defined ellipses (Stachniss et al., 2009). The new phantom, designed with TomoPhantom (Kazantsev et al., 2018), is comprised of 5 bivariate Gaussian profiles, depicting a static gas mixture, and an ellipse near one corner of the image, which serves as a reference point. This new spectral phantom can be seen in Figure 6, and a descriptive summary is provided in Table 1.

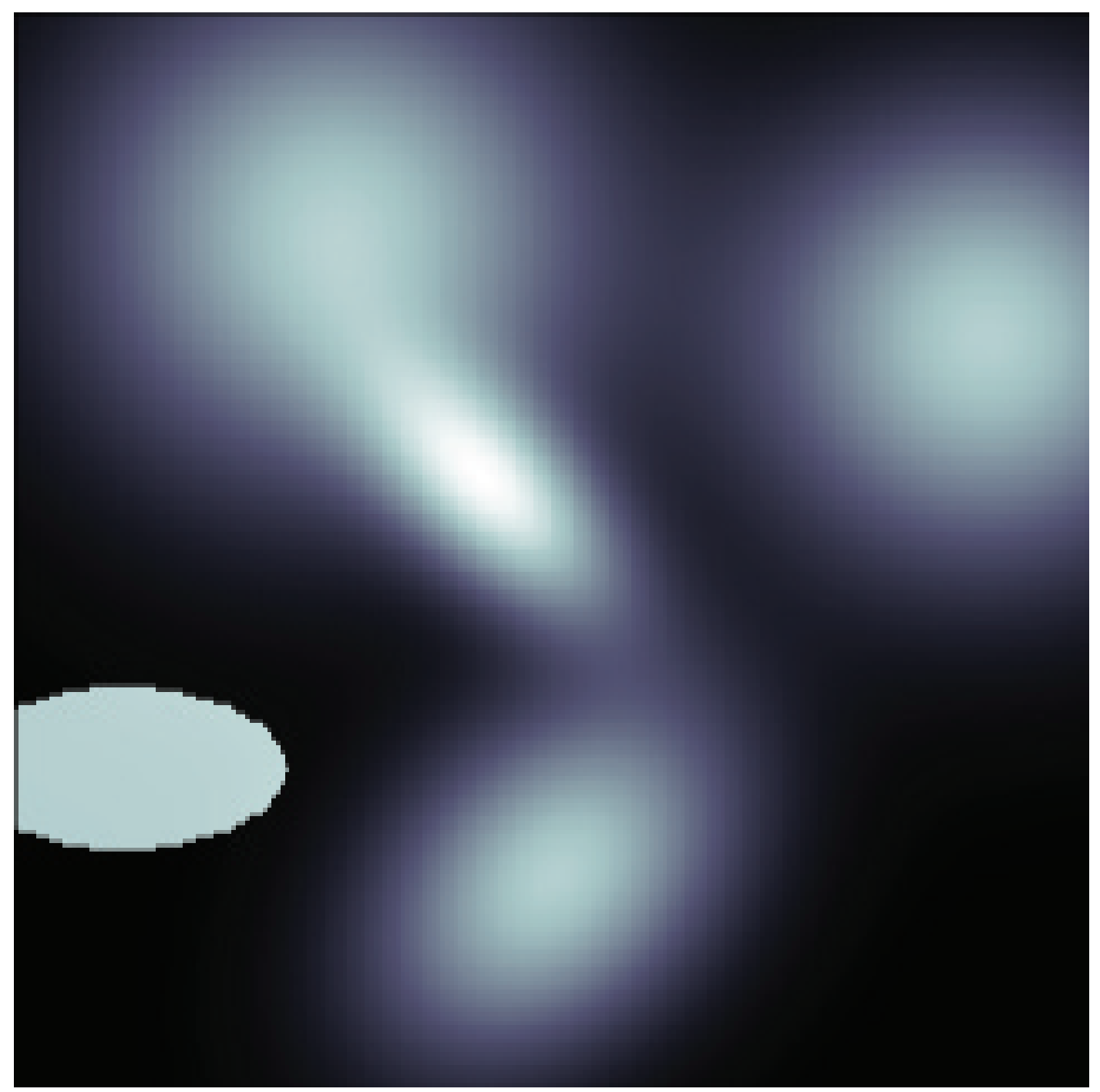

Figure 6. A graphical representation of the new spectral phantom, custom built for the TomoSim application.

5 During simulation, a phantom is totally contained within the ROI and some Gaussian noise is added to it. The phantom shares the same grid as the discretised ROI and each pixel has a value comprehended between 0 and 255 . This value is linearly correlated with the number of molecules of the target trace gases in the ROI. Software configuration allows the definition of the maximum number of molecules per pixel. Default value for $\mathrm{NO}_{2}$, the test case presented in Section 4 is 1e15 molecules. 
Table 1. Table summarising the new phantom's construction details, as a sum of 5 Gaussian profiles and an ellipse designed using TomoPhantom. In this table, $\mathrm{C} 0$ is the object's amplitude, $\mathrm{X} 0$ and $\mathrm{Y} 0$ are its center coordinates, and a and $\mathrm{b}$ are the objects half-widths. The table is constructed using TomoPhantom's particular syntax and more information can be obtained at (Kazantsev et al., 2018).

\begin{tabular}{lcccccr}
\hline Type & C0 & X0 & Y0 & a & b & Angle \\
\hline Gaussian & 1 & $-0,1$ & $-0,1$ & 0,25 & 0,5 & -45 \\
Gaussian & 1 & 0,6 & 0 & 0,65 & 0,45 & -45 \\
Gaussian & 1 & $-0,6$ & $-0,4$ & 0,8 & 0,8 & 0 \\
Gaussian & 1 & $-0,4$ & 0,8 & 0,7 & 0,7 & 0 \\
Ellipse & 1 & 0,4 & $-0,8$ & 0,3 & 0,15 & 0 \\
\hline
\end{tabular}

\subsection{Reconstruction}

Any tomographic reconstruction requires the previous and detailed knowledge of the ray geometry of the problem. This implies that the space being reconstructed is discretised, so that it can be addressed through computational routines. In this case, the discretisation consists in overlaying a 1000 x 1000 pixel grid $(1 \mathrm{~m}$ square pixels, considering a $1 \mathrm{~km}$ diameter circular drone trajectory). By applying Siddon's algorithm to this geometry, the lengths that each ray traverse in each pixel of the grid are retrieved, assembling the system matrix. The system matrix is a complete description of the problem's geometric properties, and is therefore characteristic of each experiment, depending on the angular intervals between projections ( $\Delta$ in this case) and on the size and number of the pixels in the discretisation grid.

TomoSim then performs a resorting operation on the sinogram, in order to transform the fan beam projections into parallel projections, greatly simplifying image reconstruction. Since the angular interval between the fans and the rays within the fans is the same $(\Delta)$, resorting is greatly simplified (Kak and Slaney, 2001). After discretisation and the necessary resorting steps are taken, the software reconstructs the images with three different algorithms: MLEM, FBP and SART.

Finally, after the images are calculated, the simulator must convert the pixel values back into molecule numbers. For this, the software runs Siddon algorithm on the reconstructed images for a selected number of angles. Resulting projection values are then compared with the projection values of the original images in order to find a converting parameter that allows the presentation of accurate concentration values.

\subsection{Error Estimation}

There are three major error sources in TomoSim: geometric errors, spectroscopic errors and reconstruction errors. Geometric errors come from the fact that the device's positioning has an associated error: the drone is not where it thinks it is, nor does it point to where it thinks it points. TomoSim addresses this kind of error in a Monte Carlo like fashion. Positioning and pointing errors are assumed to be normal. Each time a point is calculated, the software generates a normally distributed 0 mean random number, with a standard deviation equal to the rated error of the positioning system and sums it to the intended point (error calculations illustrated in Appendix A). Given the ratios between the linear distances involved in the trajectory 
and the positioning errors, geometric errors have a very small contribution towards the end results. On the spectroscopic level, errors come from the instruments used for capturing the data. TomoSim takes this noise into account by adding Gaussian noise spectra to each measurement, for which the magnitude is configurable via its standard deviation, a method previously followed in (Stutz and Platt, 1996). This approach is only valid insofar as the captured spectra are perfectly calibrated regarding spectral shift and squeeze, which is an acceptable assumption for a simulation. Finally, the software has to deal with the reconstruction errors. In image reconstruction from projections, it is common to use techniques such as Mean Squared Error (MSE) as a metric with which to assess the algorithm's performance. This simulator was also evaluated in this light, in two different ways. First, the MSE for the whole reconstructed image was calculated. This enables the possibility to look at the reconstruction as a whole and visually tell where it is lacking and where it is better performing. Secondly, a score was calculated according to Equation 12. In this equation, and with reference to this simulator, $f$ is the original image and $g$ is the reconstructed image.

$$
E=\sqrt{\frac{\sum|g(x, y)-f(x, y)|^{2}}{\sum|f(x, y)|^{2}}}
$$

\section{Results}

This section presents, analyses and discusses results obtained by the application of the techniques and methods described in the previous two sections, i.e., the tomographic reconstruction of the phantoms which were also presented in Section 3. All reconstructions presented were performed on a $300 \times 300$ pixels phantom, and that is also their size.

\subsection{Projection Calculations}

In TomoSim, a projection is the sum of the pixel lengths (the lengths of the rays that traverse each pixel) for each ray and for the grid mentioned in Section 3. Unlike a real life situation, the contents of the ROI are completely known and correspond to the phantoms also described in Section 3 multiplied by a given maximum number of molecules. Siddon's algorithm is used in this process, and the final results of its application are the sinogram and the system matrix. Figure 7 contains some examples of these matrices, both for the new spectral phantom and Shepp-Logan's, for two projection intervals ( $\Delta$ values), 1 and 5 degrees. Reconstruction degradation is clearly visible even in the sinogram stage.

\subsection{Reconstruction Results}

Images corresponding to the trace gas distribution within the ROI were reconstructed using iterative and analytical methods. In Figure 8, one can see the reconstruction results for the three tested methods when applied to the new spectral phantom; Figure 9 shows the graphical representation of the reconstruction errors for the spectral phantom and is accompanied by Table 2; and in Figure 11, a comparison between reconstructions with different $\Delta$ values is presented, also for the new spectral phantom. Although the new phantom was clearly privileged in this paper, mostly due to its being more similar to real life measurements, reconstruction for the Shepp-Logan phantom is displayed in Figure 10. All images displayed are normalised to the 0-1 range. 
https://doi.org/10.5194/amt-2020-26

Preprint. Discussion started: 7 May 2020

(C) Author(s) 2020. CC BY 4.0 License.
Atmospheric

Measurement

Techniques

Discussions
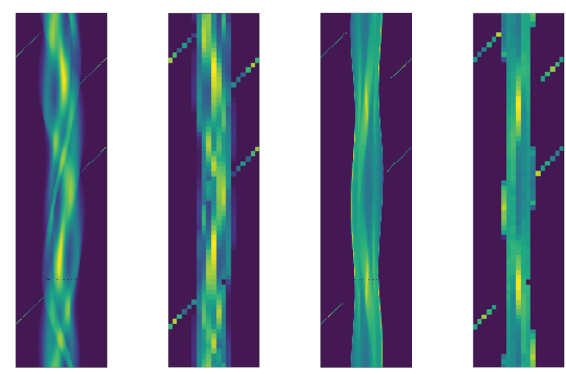

Figure 7. Sinogram examples for different projection intervals. Left to right: spectral phantom at 1 and 5 degrees, Shepp-Logan phantom at 1 and 5 degrees.
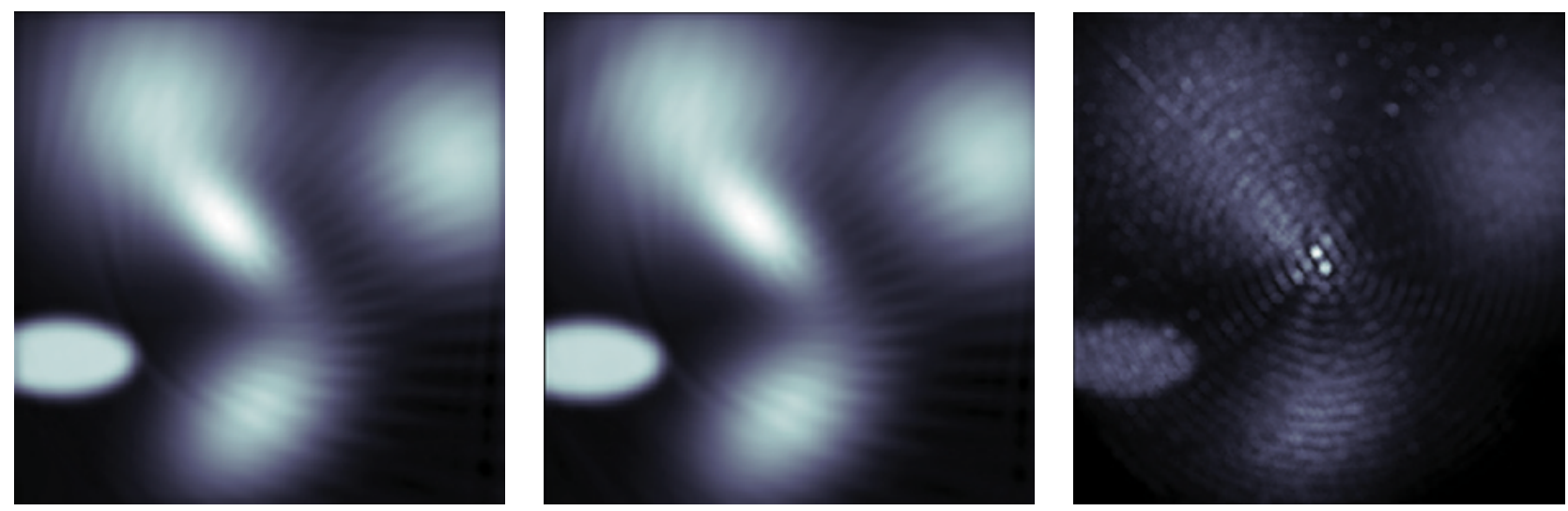

Figure 8. Tomographic reconstruction results for the spectral phantom and algorithms presented in Section 3 (left to right: FBP, SART and MLEM). The original phantom can be seen in Figure 6

\subsection{Discussion}

Results presented in the previous subsection raise a series of pertinent observations that should be addressed in discussion. The first remark goes to the fact that the number of projections used was adequate to perform the tomographic reconstruction in adequate fashion. Figure 8 is a direct example of this. This was an expected result, since there are already in the literature many examples of studies in which a considerably lower number of projections was used, still producing satisfactory results (Pundt et al., 2005). Moreover, and since the simulation software automatically includes errors in the calculations, this figure also proves that geometric error plays a very limited role in changing the result of the reconstruction. Given the difference in size between the drone's trajectory and the geometric error, this was also a predicted result.

Even with the relatively low number of projections produced by the drone's trajectory and measurement strategy, all reconstruction algorithms were able to produce a reconstructed image that resembled the phantom that generated it. However, $\Delta$, the angular interval between the stops in the trajectory was crucial, and values above 2 degrees fail to produce satisfactory images. 

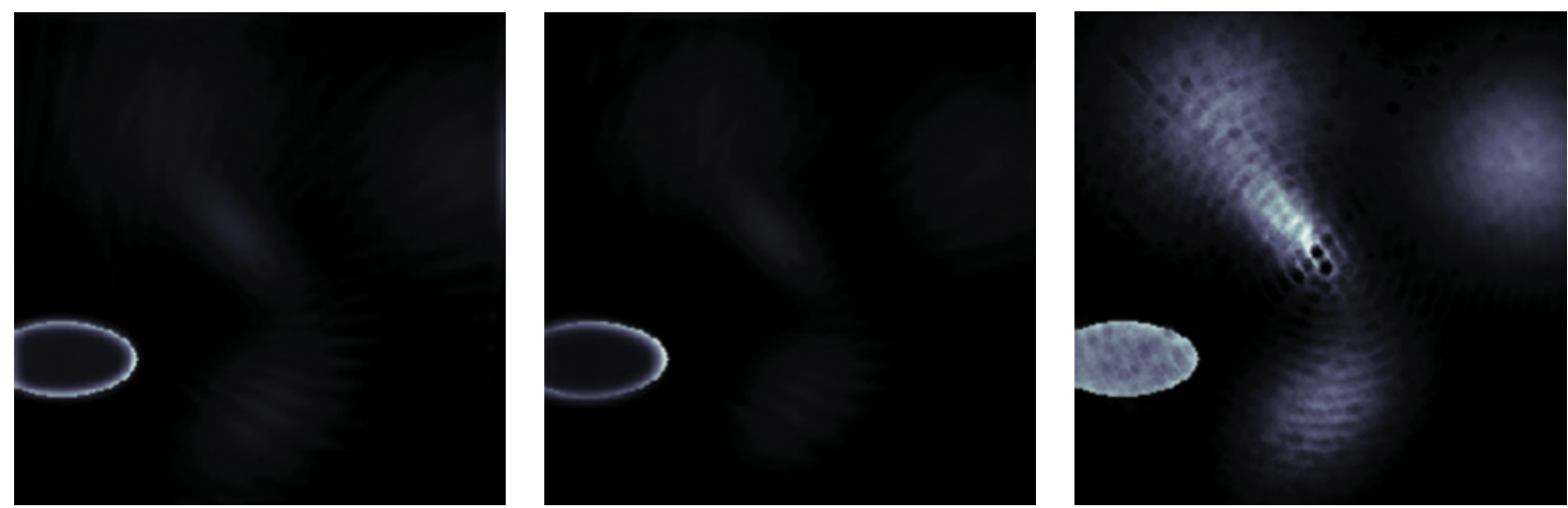

Figure 9. Tomographic reconstruction errors from reconstructions presented in Figure 8. Left to right: FBP, SART, MLEM

Table 2. Reconstruction error table for both phantoms and several projection intervals. Errors presented were calculated using the Root Mean Square Errors, normalised to the range of the reconstructed image.

\begin{tabular}{lcccc} 
& Algorithm & \multicolumn{3}{c}{ Projection Intervals (deg) } \\
\cline { 2 - 5 } & & $\mathbf{1}$ & $\mathbf{2}$ & $\mathbf{3}$ \\
\hline \multirow{3}{*}{ Spectral Phantom } & MLEM & 0.9174194 & 0.95049936 & 0.9993995 \\
\cline { 2 - 5 } & FBP & 0.2836405 & 0.26952186 & 0.2734383 \\
\cline { 2 - 5 } Shepp-Logan Phantom & SART & 0.31770208 & 0.27214736 & 0.62988657 \\
\cline { 2 - 5 } & MLEM & 0.8075044 & 0.9464826 & 0.9994179 \\
\cline { 2 - 5 } & FBP & 1.0412588 & 1.0717094 & 1.1455626 \\
\cline { 2 - 5 } & SART & 1.0422108 & 1.0802156 & 1.0248436 \\
\hline
\end{tabular}

This indicates that the simulator is working at or near the reconstruction limit for these techniques. Figure 9 and Table 2 also demonstrate this.

Before discussing reconstruction results, it is necessary to make two prior remarks: while FBP and SART were used directly from one of Python's ecosystem most used libraries, SciPy, the MLEM routine was custom coded for this application. As a result, one could expect some differences regarding the level of optimisation, which should be significantly higher on the SciPy version and reflected on the performance of both algorithms. The MLEM approach was pursued nonetheless, mainly in order to have a geometry-independent reconstruction to experiment with (technically, SART is also geometry-independent, but this particular implementation does require parallel beam conversion).

As stated in section 3.5, three different kinds of error influence the reconstruction results: geometric, spectroscopic and reconstruction errors. The first kind of error is directly included in projection calculations, through the application of a Monte 

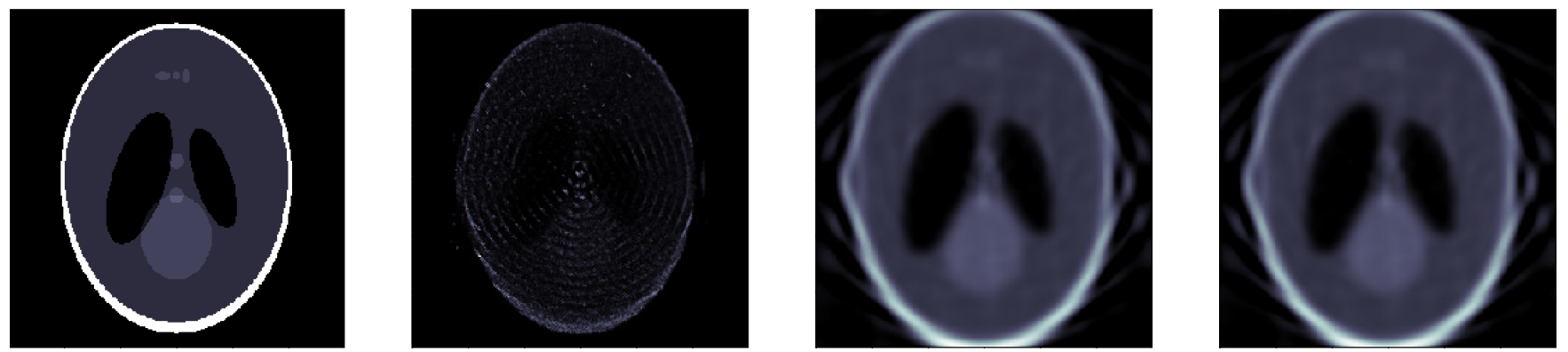

Figure 10. Shepp Logan reconstruction examples for 1 degree projection interval angle. Left to right: original phantom, MLEM, FBP and SART reconstructions.
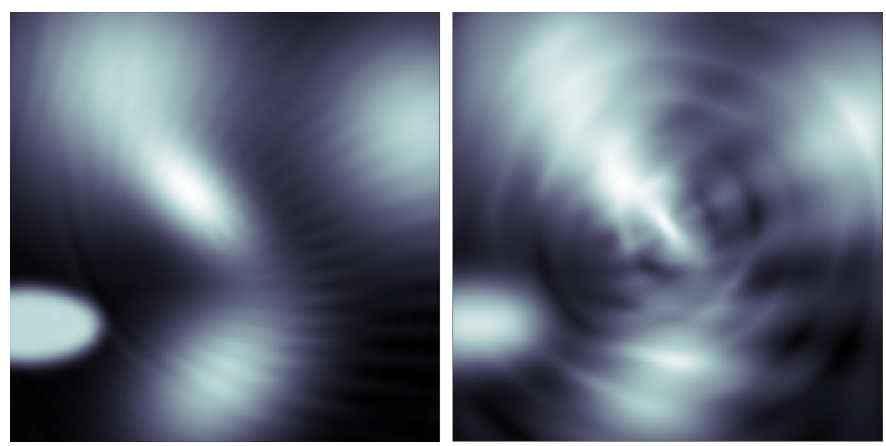

Figure 11. Reconstruction comparison for the New Phantom. On the left the reconstruction for 1 degree; on the right, for 3 degrees. Note the image degradation

Carlo-like method to the geometry described in Section A. The second kind of uncertainty comes from the spectrum acquisition process itself, which is not perfect. If one considers there are no systematic errors present in the results, which is an acceptable premise in a simulation, then these errors can be simulated by the inclusion of Gaussian noise in the spectral measurements. This approach is based on the one used in (Stutz and Platt, 1996), in which a Gaussian noise spectrum is added to the spectrum 5 of interest in order to simulate how the error behaves with a degraded signal. Finally, reconstruction errors come from the finite precision of the calculations that render the images. These errors were presented in Section 4.

The three methods were also evaluated as to how they perform computationally, by measuring the time it took to produce the images in Figure 8 using a Paperspace P4000 cloud computing instance. In this regard, the fastest method was FBP, which took around 3 seconds to reconstruct. The second was MLEM, with around 50 seconds for 1000 iterations, and finally came SART, with 1 minute and 50 seconds for 1 iteration. One relevant observation comes from the fact that MLEM was significantly faster than SART, even taking into account the difference in optimisation, which was not an expected result and may indicate some reconstruction enhancing technique on SART's side, as the literature seems to indicate that this technique is faster than MLEM (Defrise et al., 2005). 
All things considered, the FBP algorithm produces a very good reconstruction, equivalent to SART's, while being more than 10 times faster, indicating that for this kind of application and with this kind of projection information, it is the best reconstruction algorithm.

\section{Conclusions and Future Work}

5 The initial goal of the TomoSim software project was to develop a simulation platform with which to recreate the tomographic reconstruction of the column density distribution for a number of target atmoshperic trace gases.

The software program was written using the Python language and some numeric calculation libraries, such as NumPy and SciPy. Using these two libraries had two main effects: on the one hand, it enabled the programmers to easily create and manipulate matrices and vectors (images, for instance), and on the other, they greatly improved the running speed of the code, since their core is written in lower level languages (namely $\mathrm{C}$ ).

The simulations that the software performs prove that, if the final device is programmed to comply to trajectory and acquisition requirements, reconstruction is perfectly achievable, even with relatively low projection numbers (comparing with medical imaging procedures). This brings another significant conclusion which is that the devised acquisition definitions, which produce a set of fan beam arrays, provide sufficient projection information to run the reconstruction and achieve plausible results.

TomoSim runs three algorithms on the projection data in order to produce the spectral mapping of the target pollutants: FBP (analytical), SART and MLEM (both algebraic). SART offered the best results, at the expense of time. The analytical algorithm produced very nearly the same results, but took a fraction of the time when comparing with either SART of MLEM. The MLEM algorithm cannot be directly compared to the SART algorithm, due to differences in the optimisation levels of both routines, but had nonetheless a reasonable time performance altogether, although producing the poorest reconstruction results.

Regarding future developments, there are three main avenues that should be explored:

Other phantoms: Presently, TomoSim only includes tomographic reconstruction for two different phantoms. While this is sufficient for simulation, it would be desirable to have some more phantoms, which could mimic other concentration distributions of interest.

Paradigm shift: This simulation software was developed under the passive DOAS analysis model. Active measurements are much more versatile and accurate, and it would be interesting to develop this same technique using an artificial light source. Of course this would require many adaptations, namely regarding equipment and trajectory (probably even algorithms and interpolations).

Threedimensional reconstruction: TomoSim was developed to produce the reconstruction of a twodimensional image corresponding to the spatial distribution of an array of target trace gases. It would be much more interesting to have a threedimensional equivalent. As far as simulation goes, this is one of the most immediate developments for this project. On a more tangible level, the additional dimensional would make the problem much more complex, mainly because of trajectory and battery logistics. 


\section{Appendix A: Geometric calculations}

\section{A1 $\mathbf{P}_{2}$ determination}

Figure A1 is a schematic snapshot of a point in which the drone is taking a spectrum in of its stops. Here, the drone's position,

$5 \mathbf{P}_{1}$, is given by the distance $\mathrm{D}$ and the angle $\beta$, while the gimbal is pointing at a direction at an angular distance of $\gamma$ from line $0 \mathrm{P}_{1}$. Point $\mathrm{P}_{2}$, which is not known, is at the intersection between the trajectory's circumference and line $\mathrm{P}_{1} \mathrm{P}_{2}$. Parametrically, any point in this line can be expressed as $P_{1}+t \cdot\left(P_{2}-P 1\right)$, with $t$ being a scalar. Moreover, to say a point $X$ is on this circumference is the same as writing $X-Q=D$. Here, $Q$ is the centre of the trajectory and can therefore be eliminated from the equation. If one is to expand these equations, the situation can be accurately described by Equation A1.

$\left|P_{2}\right|=D^{2}$

Unravelling the expressions in Equation A1, and making use of the algebraic property that says $|A|^{2}=A \cdot A$, the expression becomes a two degree equation, as stated in Equation A2, if one writes $P_{2}-P_{1}$ as $V$.

$t^{2} V^{2}+2 \cdot V \cdot P_{1} \cdot t+P_{1}^{2}-D^{2}=0$

If line $\mathrm{P}_{1} \mathrm{P}_{2}$ non-tangentially intersects the circumference, solving Equation $\mathrm{A} 2$ renders two values for $\mathrm{t}$ (which correspond to $\mathrm{P}_{1}$ and $\mathrm{P}_{2}$ ). Selection is made by determining the returned value of $t$ that maximises the euclidean distance between the produced point and $\mathrm{P}_{1}$.

\section{A2 Geometric error determination}

Figure A2 is a graphical representation for the reasoning behind the geometric error estimation. There are two types of error in this image: the ones that come from the RTK-GPS system (positioning error, denoted $\epsilon_{p}$ ) and the ones that come from the gimbal (pointing error, denoted $\epsilon_{\gamma}$ ). TomoSim considers these errors to be normally distributed, and the two $\epsilon$ values correspond to their standard deviation. To introduce the error into the simulation, the software calculates the theoretical $\mathrm{P}_{1}$ from the $\beta$ and $D$ values (see Figure A1) and then adds a normally distributed random number that respects $\epsilon_{p}$, retrieving the true $\mathrm{P}_{1}$. This new point is used to draw the theoretical line $\mathrm{P}_{1} \mathrm{P}_{2}$ and the pointing error is added using the same process as in $\mathrm{P}_{1}$. Given the very low gimbal error, the small angles approximation $\left(\sin \theta=\theta\right.$ ) is used to determine the theoretical value of $\mathrm{P}_{2}$, on the drone's circular trajectory. Finally, the software adds again the positioning error, in the same manner as it had on $\mathrm{P}_{1}$. As a finishing remark, it is important to note that in Figure A2, all errors are extremely exaggerated as they would not be visible otherwise, due to the huge size difference between them and the trajectory. 
https://doi.org/10.5194/amt-2020-26

Preprint. Discussion started: 7 May 2020

(c) Author(s) 2020. CC BY 4.0 License.

(c) (i)
Atmospheric Measurement Techniques Discussions

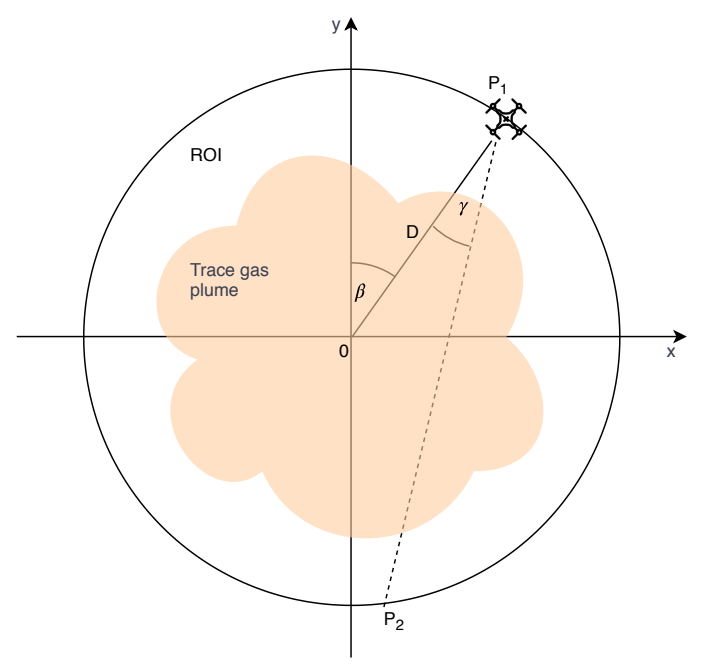

Figure A1. $\mathrm{P}_{2}$ Calculation.

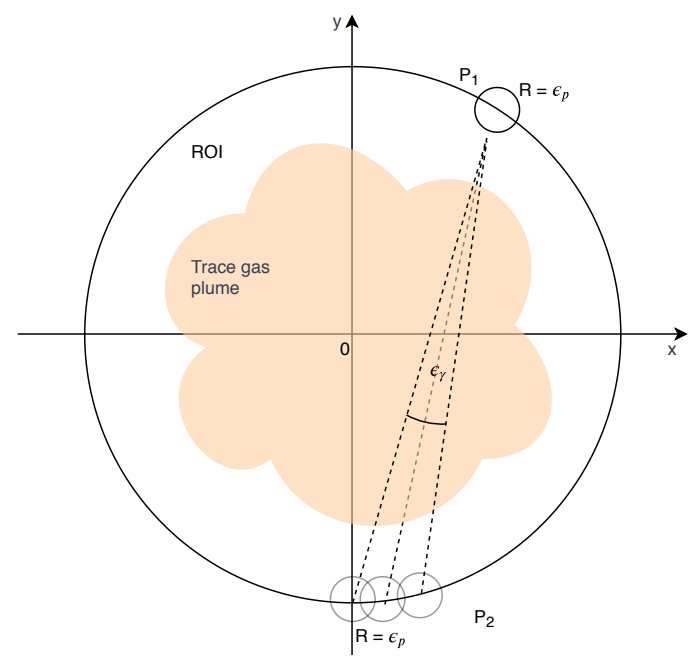

Figure A2. Error estimation graphical representation. Note errors are extremely exaggerated for visualisation purposes. 


\section{References}

Andersen, A.: Simultaneous Algebraic Reconstruction Technique (SART): A superior implementation of the ART algorithm, Ultrasonic Imaging, 6, 81-94, https://doi.org/10.1016/0161-7346(84)90008-7, https://linkinghub.elsevier.com/retrieve/pii/0161734684900087, 1984.

APS: August 15, 1758: Death of Pierre Bouguer, American Physical Society News, 20, 2, https://www.aps.org/publications/apsnews/201108/ upload/AugSept2011.pdf, 2011.

Avantes: AvaSpec-Mini: Small and powerful OEM spectrometer, https://www.avantes.com, 2019.

Bruyant, P. P.: Analytic and iterative reconstruction algorithms in SPECT., Journal of nuclear medicine : official publication, Society of Nuclear Medicine, 43, 1343-58, http://www.ncbi.nlm.nih.gov/pubmed/12368373, 2002.

Byer, R. L. and Shepp, L. A.: Two-dimensional remote air-pollution monitoring via tomography, Optics Letters, 4, 75, https://doi.org/10.1364/OL.4.000075, https://www.osapublishing.org/abstract.cfm?URI=ol-4-3-75, 1979.

Casaballe, N., Osorio, M., Di Martino, M., and Frins, E.: Comparison Between Regularized Optimization Algorithms for Tomographic Reconstruction of Plume Cross Sections in the Atmosphere, Earth and Space Science, 4, 723-736, https://doi.org/10.1002/2017EA000341, http://doi.wiley.com/10.1002/2017EA000341, 2017.

Celera: UAV Gimbal, https://www.celeramotion.com/applications/cameras-and-satcom/uav-gimbal-ssin-06/, 2019.

15 Defrise, M., Kinahan, P. E., and Michel, C. J.: Positron Emission Tomography, Springer London, London, 2005.

DJI: DJI E1200 Pro Tuned Propulsion System, 2015.

Frins, E., Bobrowski, N., Platt, U., and Wagner, T.: Tomographic multiaxis-differential optical absorption spectroscopy observations of Sun-illuminated targets: A technique providing well-defined absorption paths in the boundary layer, Applied Optics, 45, 6227-6240, https://doi.org/10.1364/AO.45.006227, 2006.

20 Gao, H.: Fast parallel algorithms for the x-ray transform and its adjoint, Medical Physics, 39, 7110-7120, https://doi.org/10.1118/1.4761867, http://doi.wiley.com/10.1118/1.4761867, 2012.

Gunderman, R.: Essential Radiology: Clinical Presentation, Pathophysiology, Imaging, 2nd ed., Thieme, 2 edn., 2006.

Herman, G. T.: Image Reconstruction From Projections, Real-Time Imaging, 1, 3-18, https://doi.org/10.1006/rtim.1995.1002, 1995.

Kak, A. and Slaney, M.: Principles of Computerized Tomographic Imaging, Society of Industrial and Applied Mathematics, 2001.

Kazantsev, D., Pickalov, V., Nagella, S., Pasca, E., and Withers, P. J.: TomoPhantom, a software package to generate 2D-4D analytical phantoms for CT image reconstruction algorithm benchmarks, SoftwareX, 7, 150-155, https://doi.org/10.1016/J.SOFTX.2018.05.003, https://www.sciencedirect.com/science/article/pii/S2352711018300335?via\{\%\}3Dihub, 2018.

Kern, C., Deutschmann, T., Vogel, L., Wöhrbach, M., Wagner, T., and Platt, U.: Radiative transfer corrections for accurate spectroscopic measurements of volcanic gas emissions, Bulletin of Volcanology, 72, 233-247, https://doi.org/10.1007/s00445-009-0313-7, 2010.

Laepple, T., Knab, V., Mettendorf, K.-U., and Pundt, I.: Longpath DOAS tomography on a motorway exhaust gas plume: numerical studies and application to data from the BAB II campaign, Atmospheric Chemistry and Physics Discussions, 4, 2435-2484, https://doi.org/10.5194/acpd-4-2435-2004, www.atmos-chem-phys.org/acp/4/1323/http://www.atmos-chem-phys-discuss. net/4/2435/2004/, 2004.

Merlaud, A.: Development and use of compact instruments for tropospheric investigations based on optical spectroscopy from mobile platforms, Phd thesis, Faculté des Sciences - Université Catholique de Louvain, Louvain, http://books.google.com/ books?hl=en $\{\&\} \mathrm{lr}=\{\&\} \mathrm{id}=\mathrm{inXVSyR} 82 \mathrm{zwC}\{\&\}$ oi=fnd $\{\&\}$ pg=PR3 $\{\&\} \mathrm{dq}=$ Development+and+use + of + compact+instruments + for + 

4G0eVEvthJXuqd8WI3IWjjVXuXc, 2013.

Oliphant, T. E.: Python for Scientific Computing, Computing in Science \& Engineering, 9, 10-20, https://doi.org/10.1109/MCSE.2007.58, http://ieeexplore.ieee.org/document/4160250/, 2007.

Omegon: MightyMak 60 \& 80, https://www.omegon.eu/telescopes/omegon-maksutov-telescope-mightymak-60/p, $46442\{\#\} \operatorname{tab}\left\{_{-}\right\} \operatorname{bar}\left\{_{-}\right\} 4\left\{_{-}\right\}$select, 2019.

Perez, F. and Granger, B. E.: IPython: A System for Interactive Scientific Computing, Computing in Science \& Engineering, 9, 21-29, https://doi.org/10.1109/MCSE.2007.53, http://ieeexplore.ieee.org/document/4160251/, 2007.

Platt, U. and Stutz, J.: Differential Optical Absorption Spectroscopy - Principles and Applications, Springer-Verlag Heidelberg Berlin, Heidelberg, Germany, 2007.

Press, W. H., Teukolsky, S. A., Vetterling, W. T., and Flannery, B. P.: Numerical Recipes: The Art of Scientific Computing, 3rd editio edn., 2007.

Pundt, I., Mettendorf, K. U., Laepple, T., Knab, V., Xie, P., Lösch, J., Friedeburg, C. V., Platt, U., and Wagner, T.: Measurements of trace gas distributions using Long-path DOAS-Tomography during the motorway campaign BAB II: Experimental setup and results for NO 2 , Atmospheric Environment, 39, 967-975, https://doi.org/10.1016/j.atmosenv.2004.07.035, 2005.

Shepp, L. A. and Logan, B. F.: The Fourier reconstruction of a head section, IEEE Transactions on Nuclear Science, 21, 21-43, https://doi.org/10.1109/TNS.1974.6499235, http://ieeexplore.iee.org/document/6499235/, 1974.

Shepp, L. A. and Vardi, Y.: Maximum Likelihood Reconstruction for Emission Tomography, IEEE Transactions on Medical Imaging, 1, 113-122, https://doi.org/10.1109/TMI.1982.4307558, https://ieeexplore.iee.org/document/4307558/, 1982.

Siddon, R. L.: Fast calculation of the exact radiological path for a three-dimensional CT array, Medical Physics, 12, 252-255, https://doi.org/10.1118/1.595715, http://doi.wiley.com/10.1118/1.595715, 1985.

Stachniss, C., Plagemann, C., and Lilienthal, A. J.: Learning gas distribution models using sparse Gaussian process mixtures, Autonomous Robots, 26, 187-202, https://doi.org/10.1007/s10514-009-9111-5, http://link.springer.com/10.1007/s10514-009-9111-5, 2009.

Struve, W. S.: Fundamentals of Molecular Spectroscopy, Wiler-Interscience, 1989.

Stutz, J. and Platt, U.: Numerical analysis and estimation of the statistical error of differential optical absorption spectroscopy measurements with least-squares methods, Applied Optics, 35, 6041, https://doi.org/10.1364/ao.35.006041, 1996.

Stutz, J., Hurlock, S. C., Colosimo, S. F., Tsai, C., Cheung, R., Festa, J., Pikelnaya, O., Alvarez, S., Flynn, J. H., Erickson, M. H., and Olaguer, E. P.: A novel dual-LED based long-path DOAS instrument for the measurement of aromatic hydrocarbons, Atmospheric Environment, 147, 121-132, https://doi.org/10.1016/j.atmosenv.2016.09.054, http://linkinghub.elsevier.com/retrieve/pii/S1352231016307713, 2016.

Triantafyllou, D. and Aspragathos, N. A.: Intelligent Robotics and Applications, vol. 7101 of Lecture Notes in Computer Science, Springer Berlin Heidelberg, Berlin, Heidelberg, https://doi.org/10.1007/978-3-642-25486-4, http://link.springer.com/10.1007/978-3-642-25486-4, 2011.

Zimmermann, F., Eling, C., Klingbeil, L., and Kuhlmann, H.: PRECISE POSITIONING of UAVS - DEALING with CHALLENGING RTKGPS MEASUREMENT CONDITIONS during AUTOMATED UAV FLIGHTS, ISPRS Annals of the Photogrammetry, Remote Sensing and Spatial Information Sciences, 4, 95-102, https://doi.org/10.5194/isprs-annals-IV-2-W3-95-2017, 2017. 